\section{Definición de fragilidad social en personas mayores: una revisión bibliográfica}

\author{
CAROL ZÚÑIGA HERNÁNDEZ ${ }^{\text {la }}$
}

\section{Social frailty among older people}

Social frailty is a seldom explored concept in older people. There is dissent if it should be considered a multidimensional syndrome or it should be limited to the physical sphere. We conducted a review aiming to develop a concept of social frailty in older people using PubMed, BVS and CINAHL databases. We found ten articles. One exposes the concept of social frailty, nine operationalize social frailty using questions or items on social behavior and two use scales or measuring instruments to classify older people as "socially frail". It is concluded that social frailty is a new concept in gerontology: there are divergences as to whether it should be considered as another dimension of frailty or as an independent concept with a predictive capacity by itself to detect risk among older people.

(Rev Med Chile 2020; 148: 1787-1795)

Key words: Aged; Frailty; Frail Elderly.

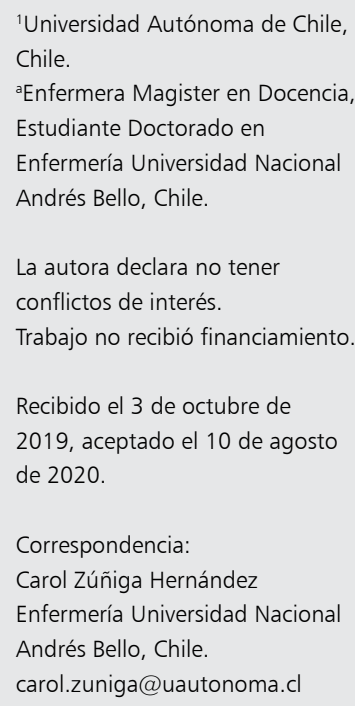

La autora declara no tener conflictos de interés.

Trabajo no recibió financiamiento.

Recibido el 3 de octubre de 2019, aceptado el 10 de agosto de 2020.

Correspondencia:

Carol Zúñiga Hernández

Enfermería Universidad Nacional

Andrés Bello, Chile.

carol.zuniga@uautonoma.cl

L a fragilidad implica una inclinación hacia la dependencia y corresponde a un estado de vulnerabilidad asociado con la edad en donde existe una disminución de las reservas funcionales en múltiples sistemas corporales aumentando la probabilidad de caídas, perdida de funcionalidad, dependencia y muerte ${ }^{1}$. Es un concepto desarrollado durante la última década del siglo recién pasado que aún permanece con ambigüedades en cuanto a sus dimensiones ${ }^{2}$. Según Linda Fried, la fragilidad corresponde a "un síndrome de disminución de las reservas y de la resistencia a estresores, resultado de un declive acumulativo a través de múltiples sistemas fisiológicos causando vulnerabilidad a resultados adversos de salud incluidos caídas, hospitalización y mortalidad" . Esta es la definición más aceptada, pero que incorpora solo los aspectos físicos de la Fragilidad. Por otra parte, autores como Iriarte y Araya explicitan otras dimensiones descritas en la literatura para este concepto, guiados particularmente según el modelo Gobbens respecto de la fragilidad en las personas mayores: la fragilidad física, psicológica y social ${ }^{4}$. Existe disenso entre estas dos corrientes respecto de si la fragilidad es un síndrome multidimensional o circunscrito al ámbito físico, por lo que la dimensión social no se encuentra desarrollada como parte de los determinantes del síndrome para las personas mayores o como un tipo de fragilidad per se. Incluso, su concepto puede ser hoy materia de discusión.

Existe escasa información respecto de la dimensión social de la fragilidad, aunque existen estudios que valoran la prevalencia de fragilidad poblacional del orden de 30 al 50\% en Latinoamérica $^{5-7}$.

El objetivo/propósito de esta revisión de la literatura es unificar criterios para desarrollar un concepto de fragilidad social en personas mayores.

\section{Material y Métodos}

Revisión Bibliográfica narrativa sobre concepto común: "Fragilidad Social en Personas Mayores" a través de análisis de contenidos y la concordancia en la definición presentada por diversos autores. 
Se realizó una búsqueda en base de datos PubMed y CINAHL en mayo 2020 con los descriptores Mesh "Aged", "Aged, 80 and over" asociados a "Social Frailty" para PubMed con resultados de 44 artículos (con la ruta de búsqueda: (social frailty[Title/Abstract]) AND ((aged[Title/Abstract])or aged, 80 and over) para la base de datos de PubMed y "Social Frailty" and "elderly or aged or older or elder or geriatric" para base de datos de CINAHL, reportándose 39 artículos, utilizando para ambas bases los operadores booleanos AND y OR. Se agrego la base de datos Biblioteca Virtual en Salud (BVS) en español utilizando los descriptores "Fragilidad" and "Social" and "Personas mayores" or "ancianos" hallándose 3 artículos asociados. Luego se aplico filtro de años de antigüedad a todos poniendo limite en 10 años desde la publicación y para estudios con seres humanos obteniéndose ningún cambio en los resultados con 86 títulos en total. A continuación, se realizo una revisión manual donde se seleccionan todos los artículos que en su título o abstract contengan el binomio "fragilidad social" (palabras juntas) o un concepto relacionado (Fragilidad multidimensional) quitándose los que se repiten en bases de datos y finalmente se obtienen 14 artículos (Figura 1).

Se utilizo como criterio de inclusión incorporar todos los artículos de cualquier tipo que incluyeran en desarrollo del documento una definición de fragilidad social y que el artículo se encuentre escrito en español o inglés.

Finalmente se realizó una depuración en base a criterios de inclusión y se obtienen 12 documentos para analizar (Tabla 1).

Los resultados obtenidos se analizaron en base a su contenido, buscando en estos artículos una definición de fragilidad social.

\section{Resultados}

Se observa que de los 12 artículos seleccionados, solo 1 muestra aproximaciones conceptuales de fragilidad social. De los restantes 11 estudios 9 operacionalizan la fragilidad social usando preguntas o ítems sobre comportamiento social sin definirla conceptualmente, mientras que 2 utilizan escalas o instrumentos de medición de la esfera social para catalogar a las personas mayores de "frágiles sociales".

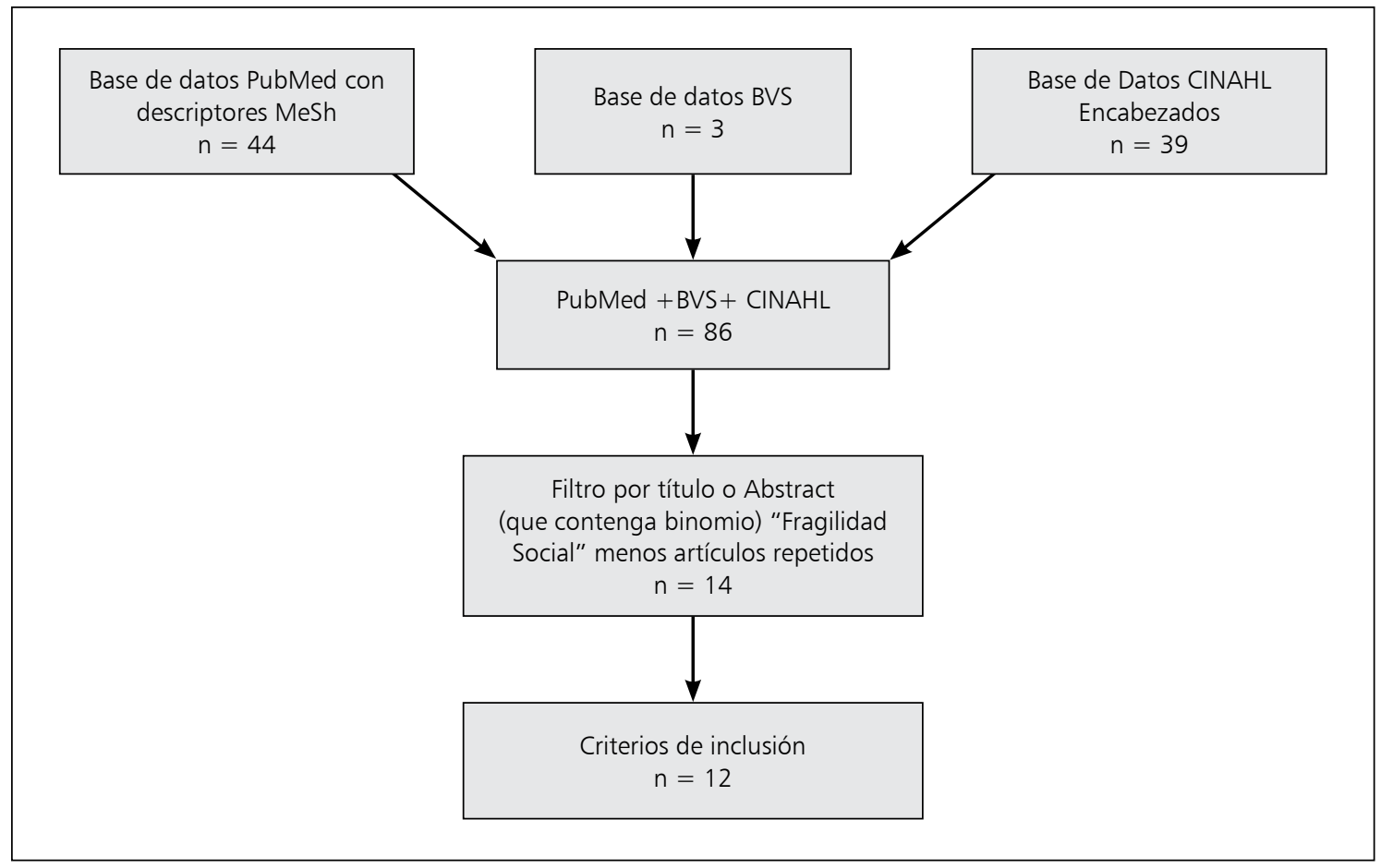

Figura 1. Flujograma de resultados. 


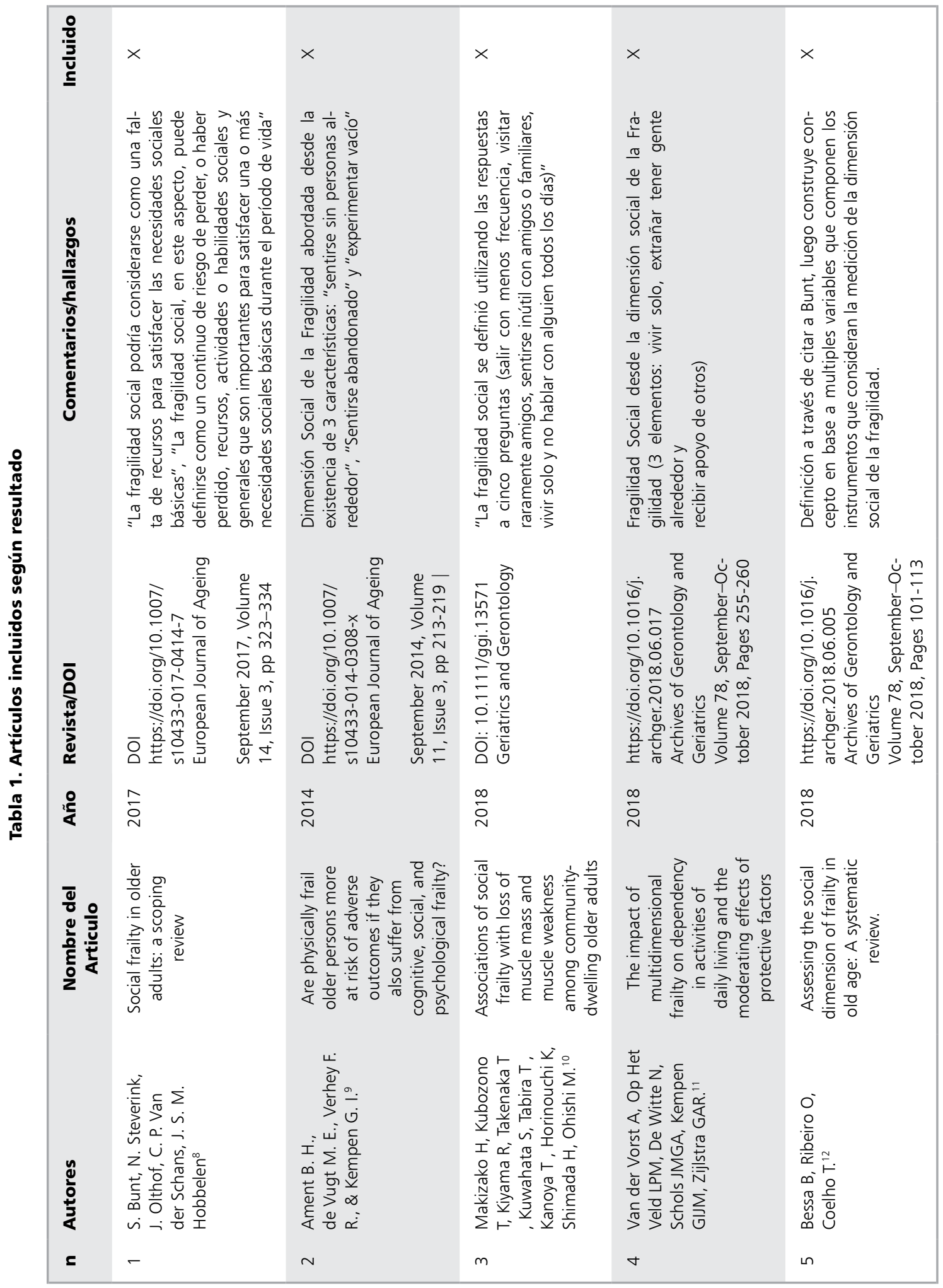




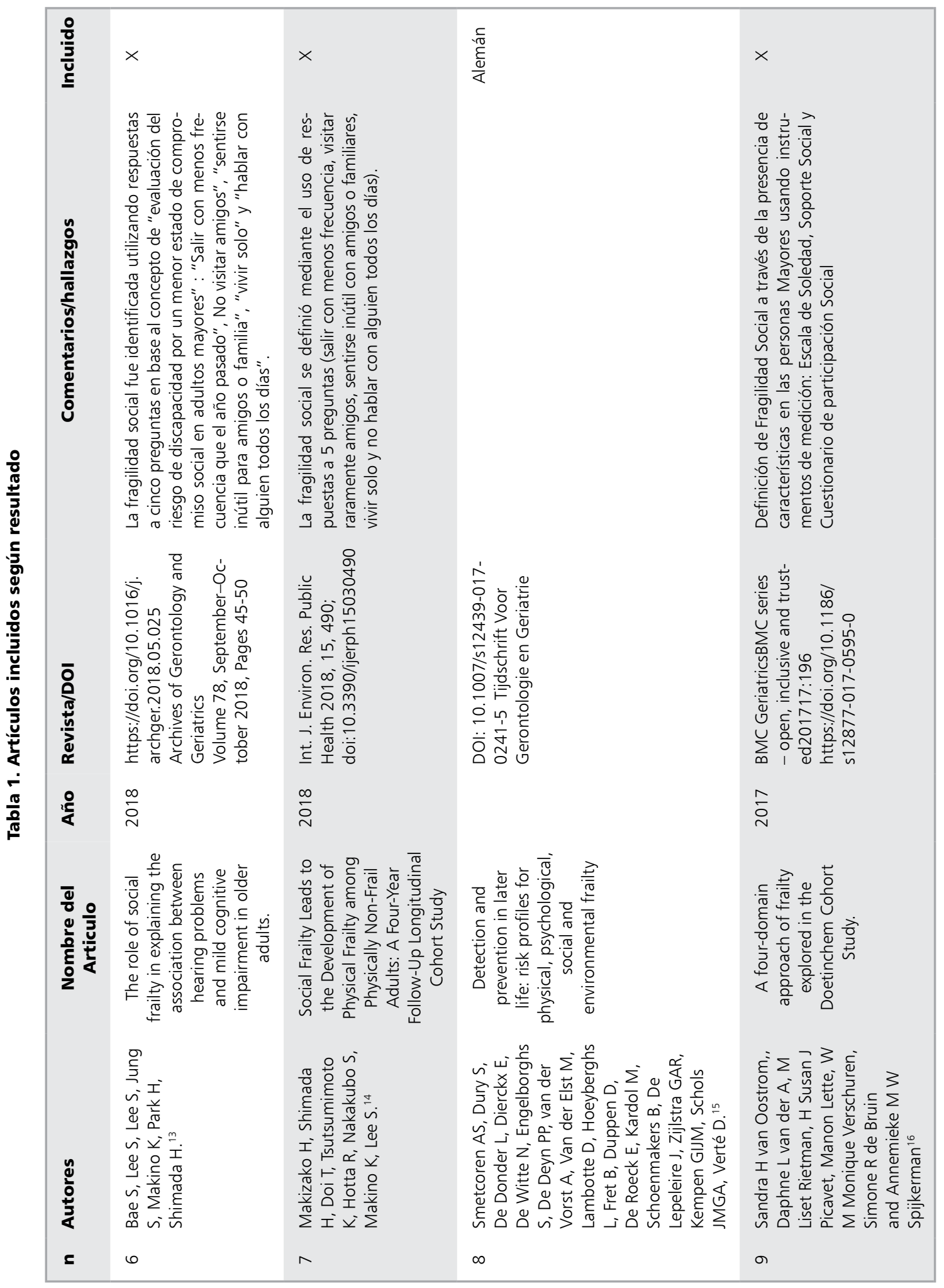




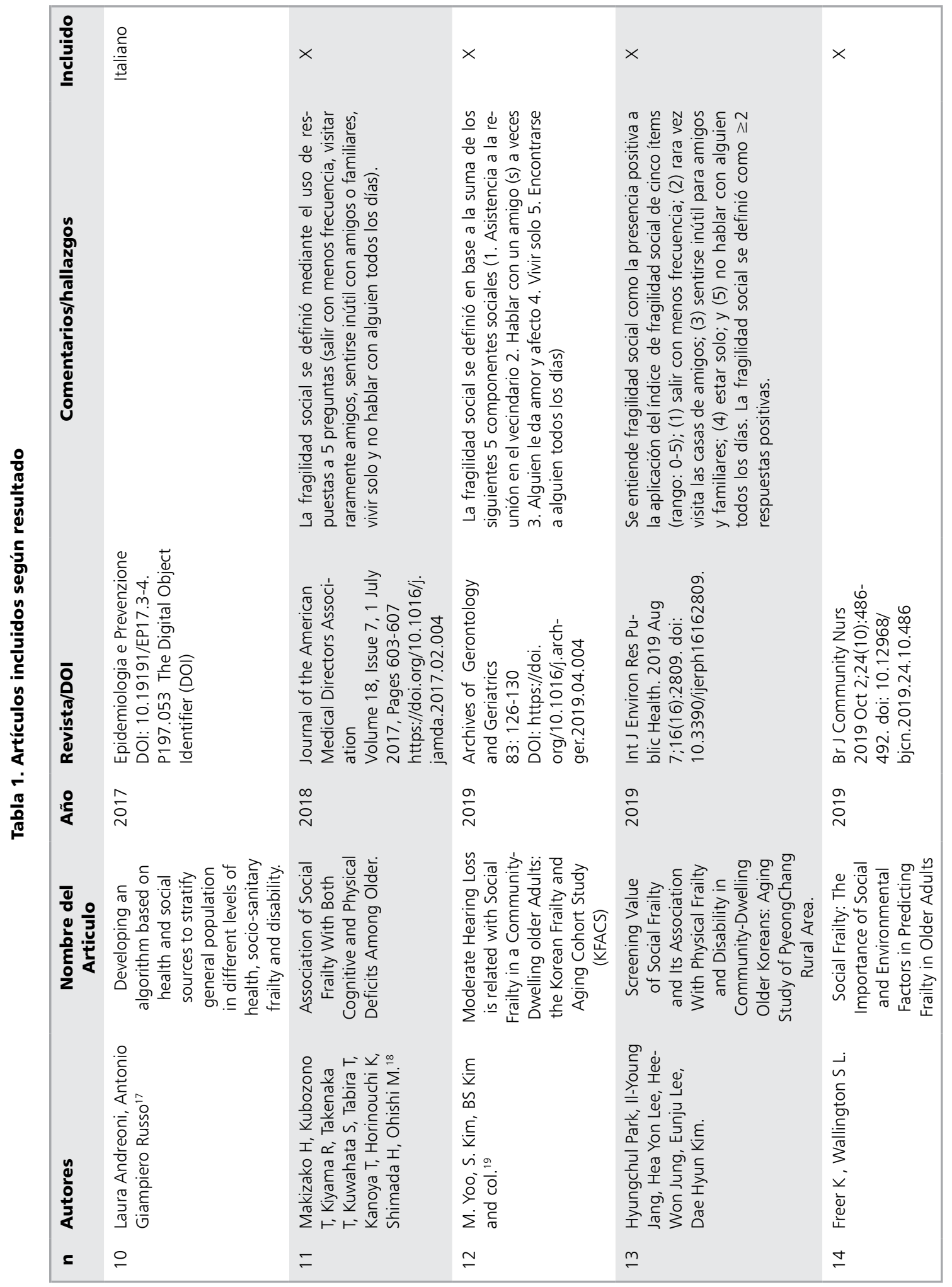


Tabla 2. Frecuencia de aparición de preguntas para evaluar fragilidad social en personas mayores

\begin{tabular}{|rll|}
\hline & Preguntas o Ítems & $\begin{array}{c}\text { Orden de Frecuencia de Aparición en la } \\
\text { evaluación de Fragilidad Social }\end{array}$ \\
\hline 2 & Salir con menos frecuencia que en el año pasado & $1^{\circ}$ \\
\hline 3 & Vivir solo & $1^{\circ}$ \\
\hline 4 & Hablar con alguien todos los días & $1^{\circ}$ \\
\hline 5 & Sentirse útil para amigos o familia & $2^{\circ}$ \\
\hline 6 & Aburrirse & $2^{\circ}$ \\
\hline 7 & Tener amigos con quien hablar por teléfono & $3^{\circ}$ \\
\hline 8 & Extrañar tener gente alrededor & $3^{\circ}$ \\
\hline 9 & Recibir ayuda de otros & $4^{\circ}$ \\
\hline 10 & Sentirse abandonado & $4^{\circ}$ \\
\hline 11 & Experimentar vacío & $5^{\circ}$ \\
\hline
\end{tabular}

\section{a) Definición de fragilidad social}

La definición de fragilidad social incluida particularmente por el articulo desarrollo por Bunt y colaboradores ${ }^{8}$ como resultado de su revisión de alcance sobre el mismo concepto, alude a la situación en la que una persona mayor se encuentra sin recursos para satisfacer sus necesidades sociales básicas: es decir "un continuo de riesgo de perder, o haber perdido recursos, actividades o habilidades sociales y generales que son importantes para satisfacer una o más necesidades sociales básicas durante el período de vida”.

\section{b) Variables que componen la fragilidad social}

Dentro de los estudios que utilizan preguntas para definir esta dimensión de la fragilidad, se observa que las que más se repiten guardan relación con la situación de convivencia con otras personas, hablar con alguien todos los días y salir con menos frecuencia que antes (Tabla 2). Las preguntas solo son enunciadas, sin referencias a la forma de medición de las respuestas.

Otros estudios utilizan instrumentos como escalas de evaluación social utilizadas para definir a una persona mayor como frágil socialmente, siendo la Escala de Soledad, el Listado de Soporte y el Cuestionario sociales de participación los que se repiten en los artículos seleccionados. Los conceptos que cubren fragilidad social sin preguntas específicas corresponden a Aislamiento
Social, Soledad, Redes Sociales, Soporte Social y Participación Social.

\section{Discusión}

La Fragilidad Social es un concepto nuevo dentro del conocimiento sobre personas mayores, aunque existen divergencias respecto de si debe considerarse como una dimensión de la fragilidad o como un concepto independiente con capacidad predictiva de daño por sí mismo.

Solo un artículo ofrece una definición de fragilidad social ${ }^{8}$, mientras que el resto de la evidencia analizada solo da variables que pueden contribuir a la definición del concepto, sin hacer una definición del mismo. El estado del avance de la fragilidad social se encuentra en una etapa de definición de concepto y de la operacionalización a través de variables que la componen.

Las preguntas, variables y características que más se repiten a la hora de definir la fragilidad social se enmarcan en la situación de compañía o de vida social de la persona mayor y no en su funcionalidad social, lo que implica una reducción de fragilidad social a aspectos puntuales del funcionamiento social de la persona mayor .Por otro lado hay autores que evalúan fragilidad social con instrumentos que miden: aislamiento social, soledad, redes sociales, soporte social y participa- 
ción social. Si bien esta forma de evaluar fragilidad social es completa y aborda más elementos que la pregunta única, plantea la dificultad de diferenciar el concepto de fragilidad social con soledad o redes de apoyo (entre otros) de forma de no estar confundiendo conceptos ya existentes.

Una aproximación para conceptualizar la fragilidad social puede venir desde la Teoría de la Función de Producción Social ${ }^{20,21}$ : esta teoría social propone que las personas tienen como metas deseables el bienestar físico y el bienestar social y que, para alcanzarlos, existen objetivos instrumentales como el estímulo, la comodidad, el status, la reafirmación de comportamiento y el sentirse querido. La idea central de esta teoría es que las personas eligen y sustituyen objetivos instrumentales para optimizar la producción de su bienestar, sujeto a restricciones en los medios de producción disponibles (limitaciones propias o externas). Esto es absolutamente aplicable al hallazgo de conceptualización de fragilidad social de la literatura revisada, ya que en las variables descritas por los diferentes autores para la medición de esta dimensión de la fragilidad están implícitos los objetivos instrumentales (sentirse útil para amigos o familia, aburrirse, extrañar tener gente alrededor, por ejemplo) y se asume que las personas mayores se encuentran sin reservas sociales para alcanzar estas metas propuestas.

La fragilidad social parece no ser vista aun como un concepto diferente al de la fragilidad física o la fragilidad "en general". Esto guarda directa relación con lo incipiente de los estudios que enfocan a la fragilidad multidimensional como relevante e independiente de los aspectos físicos del ser humano. Las determinantes sociales que conforman el estado de salud de las personas mayores, sumado al resultado del curso de vida del mismo pueden condicionar el riesgo de presentarse sin recursos frente a una condición o enfermedad física, aun cuando no se observen patrones biológicos que permitan predecir esto ${ }^{22}$. Esto equivale a decir que la falta de "capital social" de una persona mayor podría situarlo en desventaja con un par con mayores recursos para hacer frente a la misma situación de daño o enfermedad ${ }^{23}$.

Las limitaciones del desarrollo de esta revisión corresponden a que, aunque la estrategia de búsqueda incluyó los últimos 10 años, el concepto de fragilidad social aparece con más fuerza después del año 2015 y en estudios japoneses con mayor frecuencia, lo que dificulta tener evidencia necesaria para llegar a una definición e identificación de variables del concepto.

Finalmente, hasta antes del año 2015 no figura en la literatura el concepto de fragilidad social ni como una dimensión independiente ni como parte de la fragilidad multidimensional explorada por Gobbens. Los estudios previos muestran un desarrollo de la literatura respecto de aspectos de vulnerabilidad social, actividad social o aspectos sociales relacionados con la fragilidad que influyen en la posibilidad de que un individuo no tenga los recursos biológicos (físicos y mentales) suficientes para hacer frente a situaciones de estrés o franca enfermedad, Con todo, la fragilidad social puede entenderse como una situación de vulnerabilidad o riesgo de daño físico, sicológico o emocional, asociada al envejecimiento y que está determinada por la falta de recursos sociales o el bajo desarrollo de estos que son necesarios para satisfacer la necesidad de productividad social.

\section{Conclusión}

El hecho de que el concepto de fragilidad social este asociado a variables o características de las personas mayores puede indicar a) homologación de la fragilidad social con fragilidad física y b) los elementos convergentes responden a la aproximación a la fragilidad social por parte de los autores vista desde teorías sociales del envejecimiento, como la Teoría de la Función de Producción Social. Evidentemente existen otras teorías sociales que pueden soportar estas afirmaciones, pero en general el trasfondo al que apuntan guarda relacion con el "capital social" de una persona mayor $^{24}$. Si bien ya se observa el ingreso del concepto de fragilidad social en la literatura, aun no queda claro la importancia de este concepto en la predicción de discapacidad o la eventual magnitud de esta dimensión en la perdida de funcionalidad en este grupo etario.

No existe una definición consensuada respecto de la fragilidad social dado que es entendida como una dimensión de la fragilidad en general, siendo vista desde el modelo multidimensional de la misma. Se muestra en esta revisión la potencia del concepto ya no desde la multidimensionalidad de la fragilidad, sino como un concepto independiente a desarrollar y comprender, generando 
una nueva definición. Esta a su vez busca además formar parte de los elementos a considerar en la evaluación integral de las personas mayores y por qué no, de la revisión de su historia, curso de vida y relaciones sociales actuales en busca de potenciar aspectos deficitarios mejorando así la calidad de vida del individuo, disminuyendo los riegos asociados a la fragilidad. Es precisamente lo que esta revisión invita a estudiar a través de develar el concepto de fragilidad social en personas mayores.

\section{Referencias}

1. Tapia C, Valdivia Y, Varela H, Carmona A, Iturra V, Jorquera M. Indicadores de Fragilidad en adultos mayores del sistema público de salud de la ciudad de Antofagasta. Rev Med Chile 2015; 143 (4). http://dx.doi.org/10.4067/ S0034-98872015000400007

2. Freitag S, Schmidt S, Gobbens R. "Tilburg frailty indicator." Zeitschrift für Gerontologie und Geriatrie 2016; 49: 86-93. DOI: 10.1007/s00391-015-0889-9.

3. Fried LP, Tangen CM, Walston J, Newman AB, Hirsch C, Gottdiener J, et al. Cardiovascular Health Study Collaborative Research Group. Frailty in older adults: evidence for a phenotype. J Gerontol A Biol Sci Med Sci 2001; 56 (3): M146-56. doi: 10.1093/ gerona/56.3.m146.

4. Iriarte E, Araya A. Criterios de Fragilidad en Personas Mayores que Viven en una Comunidad: una Revisión de la Literatura. Rev Med Chile 2016; 144 (11). http:// dx.doi.org/10.4067/S0034-98872016001100010

5. Alvarado, Chaman, José Armando Rafael. Prevalencia y características del síndrome de fragilidad en adultos mayores en el distrito de Trujillo. Journals of gerontology 2014; 63 (12). https://doi.org/10.1093/gerona/63.12.1399.

6. Carneiro JA, Cardoso R, Durães M, Guedes M, Santos F, Costa F, et al. Frailty in the elderly: prevalence and associated factors. Rev Bras Enferm 2017; 70 (4): 747-52. http://dx.doi.org/10.1590/0034-7167-2016-0633

7. Alvarado B, Zunzunegui M, Francois Béland J. Curso de vida Condiciones sociales y de salud relacionadas con la fragilidad en hombres y mujeres mayores de América Latina. The Journals of Gerontology: Serie A 2008; 63 (12): 1399-406. https://doi.org/10.1093/ gerona / 63.12.1399.

8. Bunt S, Steverink N, Olthof J, van der Schans CP, Hobbelen JSM. Social frailty in older adults: a scoping review. European Journal of Ageing 2017;14 (3): 323-34 https://doi.org/10.1007/s10433-017-0414-7.

9. Ament BHL, de Vugt ME, Verhey FRJ, Kempen GIJM. Are physically frail older persons more at risk of adverse outcomes if they also suffer from cognitive, social, and psychological frailty? Eur J Ageing 2014; 11 (3): 213-9. https://doi.org/10.1007/s10433-014-0308-x

10. Makizako H, Kubozono T, Kiyama R, Takenaka T, Kuwahata S, Tabira T, et al. Associations of social frailty with loss of muscle mass and muscle weakness among community dwelling older adults. Geriatrics and Gerontology 2018 DOI: 10.1111/ggi.13571

11. Van der Vorst A, Op Het Veld LPM, De Witte N, Schols JMGA, Kempen GIJM, Zijlstra GAR. The impact of multidimensional frailty on dependency in activities of daily living and the moderating effects of protective factors. Archives of Gerontology and Geriatrics 2018; 78: 255-60. https://doi.org/10.1016/j.archger.2018.06.017

12. Bessa B, Ribeiro O, Coelho T. Assessing the social dimension of frailty in old age: A systematic review. Arch Gerontol Geriatr 2018; 78: 101-13. doi: 10.1016/j. archger.2018.06.005.

13. Bae S, Lee S, Lee S, Jung S, Makino K, Park H, et al. The role of social frailty in explaining the association between hearing problems and mild cognitive impairment in older adults. Archives of Gerontology and Geriatrics 2018; 78: 45-50 https://doi.org/10.1016/j. archger.2018.05.025

14. Makizako H, Shimada H, Doi T, Tsutsumimoto K, Hotta R, Nakakubo S, et al. Social Frailty Leads to the Development of Physical Frailty among Physically Non-Frail Adults: A Four-Year Follow-Up Longitudinal Cohort Study. Int. J. Environ. Res. Public Health 2018; 15: 490. DOI:10.3390/ijerph15030490

15. Smetcoren AS, Dury S, De Donder L, Dierckx E, De Witte N, Engelborghs S, et al. Detection and prevention in later life: risk profiles for physical, psychological, social and environmental frailty. Tijdschrift Voor Gerontologie en Geriatrie. 2018. DOI: 10.1007/s12439-017-0241-5

16. Oostrom S, Van der A, Rietman M, Picavet S, Lette M, Verschuren $\mathrm{M}$, et al. A four-domain approach of frailty explored in the Doetinchem Cohort Study. Geriatrics BMC series 2017; 17: 196. https://doi.org/10.1186/ s12877-017-0595-0

17. Andreoni L, Giampiero A. Developing an algorithm based on health and social sources to stratify general population in different levels of health, socio-sanitary frailty and disability. Epidemiologia e Prevenzione 2017; 3-4. P197.053. DOI: 10.19191/EP17

18. Makizako H, Kubozono T, Kiyama R, Takenaka T, Kuwahata S, Tabira T, et al. Association of Social Frailty With Both Cognitive and Physical Deficits Among 
Older. Journal of the American Medical Directors Association 2017; 18 (7): 603-7. https://doi.org/10.1016/j. jamda.2017.02.004

19. Yoo M, Kim S, Kim BS, Yoo J, Lee S, Jang HC, et al. Moderate hearing loss is related with social frailty in a community-dwelling older adults: The Korean Frailty and Aging Cohort Study (KFACS). Arch Gerontol Geriatr 2019; 83: 126-30. doi: 10.1016/j.archger.2019.04.004. Epub 2019 Apr 12. PMID: 31003135.

20. Nieboer A, Cramm J. How do older people achieve well-being? Validation of the Social Production Function Instrument for the level of well-being-short (SPF-ILs). Social Science \& Medicine 2018; 11: 304-13. https://doi. org/10.1016/j.socscimed.2018.06.036

21. Sproten A, Schwieren C. Age differences in the reaction to incentives - A test of the successful ageing extension of Social Production Functions Theory. The Journal of the Economics of Ageing 2015; 6: 176-86, ISSN 2212 828X. https://doi.org/10.1016/j.jeoa.2015.02.002.

22. Hyungchul Park, Il-Young Jang, Hea Yon Lee, Hee-Won Jung, Eunju Lee, Dae Hyun Kim. Screening Value of Social Frailty and Its Association With Physical Frailty and Disability in Community-Dwelling Older Koreans: Aging Study of PyeongChang Rural Area. Int J Environ Res Public Health 2019; 16 (16): 2809. doi: 10.3390/ ijerph16162809.

23. Freer K, Wallington SL. Social Frailty: The Importance of Social and Environmental Factors in Predicting Frailty in Older Adults. Br J Community Nurs 2019; 24 (10): 486-92. doi: 10.12968/bjcn.2019.24.10.486. 